\title{
Complete Clinical Response after Induction Chemotherapy Followed by Chemoradiotherapy in Nasopharyngeal Carcinoma: Impact on Oncologic Outcomes
}

\author{
Letaief $\mathrm{F}^{1,2}$, Berrazaga $\mathrm{Y}^{\star 1,2}$, Ayadi $\mathrm{M}^{1,2}$, Meddeb $\mathrm{K}^{1,2}$, Mokrani $\mathrm{A}^{1,2}$, Yahyaoui $\mathrm{Y}^{1,2}$, Chraiet $\mathrm{N}^{1,2}$, Rais $\mathrm{H}^{1,2}$, \\ and Mezlini $\mathrm{A}^{1,2}$
}

${ }^{1}$ Department of medical oncology, Salah Azaiz Institute, Tunis, Tunisia

${ }^{2}$ Faculty of medicine of Tunis, Tunisia

*Corresponding author: Berrazaga Y, Department of medical oncology, Salah Azaiz Institute, Tunis, Tunisia, Faculty of medicine of Tunis, Tunisia, Tel: +21694538857, E-mail: Yosra.berrazaga@yahoo.fr

Citation: Letaief F, Berrazaga Y, Ayadi M, Meddeb K, Mokrani A, et al. (2017) Complete Clinical Response after Induction Chemotherapy Followed by Chemoradiotherapy in Nasopharyngeal Carcinoma: Impact on Oncologic Outcomes. J Cancer Sci Clin Oncol 4(2): 203. doi: 10.15744/2394-6520.4.203

Received Date: February 23, 2017 Accepted Date: December 15, 2017 Published Date: December 18, 2017

\begin{abstract}
Objective: Concomitant chemoradiation (RCT) represents the standard of care for locally-advanced nasopharyngeal carcinoma (NPC).Nevertheless induction chemotherapy (IC) followed by RCT is currently an attractive approach. Some trials showed a survival benefit of this therapeutic strategy. The aim of this study was to analyze complete clinical response (CCR) after IC and to assess its impact on disease control and survival.
\end{abstract}

Materials and Methods: From January 2008 to December 2014, 40 patients with locally advanced NPC were treated in our institute. All patients received IC with fluorouracyl-cisplatin (5 FU-CDDP) or fluorouracyl-cisplatin-Docetaxel (TPF) or Adriamycin-cisplatin (AD-CDDP). After IC, clinical response was evaluated, CCR was defined by a normal clinical and computed tomography examination. After IC, 14 patients received RCT and 22 patients received radiotherapy (RT) alone.

Results: Our study included 25 men and 15 women with a median age of 41 years. Tumor was classified T1 in 5\% of patients, T2 in $27 \%$, T3 in $20 \%$ and T4 in $48 \% .80 \%$ of patients had involved nodes $\left(\mathrm{N}^{+}\right)$. Twenty patients received 5FU-CDDP, 16 received TPF and 4 received $\mathrm{AD}-\mathrm{CDDP}$. The occurrence of leucopenia was higher in the 5FU-CDDP $(\mathrm{p}<0.05)$ group. Gastrointestinal toxicity was higher in the TPF group $(\mathrm{p}<0.05)$. Anemia and thrombopenia were similar in the three groups. After IC, 18 patients $(45 \%)$ achieved CCR, 7 of them had RCT and 11 had RT alone. 21 patients (52\%) achieved partial clinical response (PR) and 1 patient developed metastases. The CCR was higher in (5FU-CDDP) group ( $>0.05$ ). CCR followed by RCT was associated to better local control than RT alone. However, there was no benefit of overall survival in the CCR group compared to partial clinical reponse (PR).

Conclusion: Complete clinical response after IC followed by RCT in locally-advanced nasopharyngeal carcinoma is associated to a better local disease control without impact on survival.

Keywords: Induction chemotherapy; Radiotherapy; Concurrent chemoradiotherapy; Complete clinical response

\section{Introduction}

Nasopharyngeal carcinomas (NPC) are mainly represented by undifferentiated carcinoma nasopharyngeal type (UCNT). NPC is a cancer with an extremely unbalanced geographical distribution. There are three levels of incidence: high 15-30 cases/100,000 inhabitants (Southern China, South East Asia), low 0.5-1/100000 inhabitants (Northern Europe, USA, Japan) and intermediate 3-12/100000 inhabitants in the Mediterranean Basin and North Africa. Tunisia has an intermediate incidence of NPC. EpsteinBarr virus (EBV) is correlated to NPC. This cancer has substantial responsiveness to both radiotherapy and chemotherapy [1,2].

To decrease the rate of locoregional failure and the risk of distant metastases, treatment has substantially evolved in the last two decades from surgery through RT to multimodal chemoradiotherapy (RCT).Current standard therapy for nasopharyngeal carcinoma (NPC) is platinum-based concurrent chemoradiation based on randomized data. However, conflicting data exist to support the addition of induction chemotherapy to RCT [3-5].

The aims of our retrospectivestudy are to analyze the outcomes of a series of patients with locally advanced nasopharyngeal carcinoma (LA-NPC) treated with platinum based IC followed by RCT or RT, to evaluate the efficacy andtoxicity of this regimen, and to study the prognostic value ofcomplete clinical response for outcomes. 


\section{Materials and Methods}

From January 2008 to December 2014, 40 patients with newly diagnosed histologically confirmed locally advanced NPC were treated in SALAH AZAIZ institute of oncology of Tunis, Tunisia. Baseline imaging included bone scan, chest X-ray, abdominal ultrasound, head and neck contrast-enhanced computed tomography Patients were staged according to the American Joint Committee on Cancer TNM 2010 ( $7^{\text {th }}$ edition). All tumors were classified T3-T4 and/or $\mathrm{N}^{+}$. All patients received induction chemotherapy. Three induction chemotherapy regimens were used: fluorouracyl-cisplatin (5 FU-CDDP), fluorouracylcisplatin-Docetaxel (TPF) with primary prophylaxis with granulocyte colony-stimulating factor (G-CSF) and Adriamycincisplatin (AD-CDDP). Schedules of induction chemotherapy are summarized in Table 1.

\begin{tabular}{|c|c|}
\hline IC schedules & Dose \\
\hline Fluorouracyl-cisplatin (5 FU-CDDP) & $\begin{array}{c}\text { Cisplatin } 80 \mathrm{mg} / \mathrm{m}^{2} \text { on day } 1,5 \text {-fluorouracil } 1,000 \\
\mathrm{mg} / \mathrm{m}^{2} \text { as a continuous infusion on days } 1-5 \\
\mathrm{JI}=\mathrm{J} 21\end{array}$ \\
\hline Fluorouracyl-cisplatin-Docetaxel (TPF) & $\begin{array}{c}\text { Docetaxel } 70 \mathrm{mg} / \mathrm{m}^{2} \mathrm{IV} \text { on day } 1 \text { plus cisplatin } 75 \\
\mathrm{mg} / \mathrm{m}^{2} \mathrm{IV} \text { on day } 1 \text { plus } 5-\mathrm{FU} 1000 \mathrm{mg} / \mathrm{m}^{2} / \text { day by } \\
\text { continuous IV infusion on days } 1-4 \\
\mathrm{JI}=\mathrm{J} 21\end{array}$ \\
\hline Adriamycin-cisplatin (AD-CDDP) & $\begin{array}{l}\text { Adriamycin }\left(60 \mathrm{mg} / \mathrm{m}^{2}\right) \text { on day } 1 \text {-cisplatine }(100 \mathrm{mg} / \\
\left.\mathrm{m}^{2}\right) \text { on day1 JI=J21 }\end{array}$ \\
\hline
\end{tabular}

Table 1: Schedules of Induction Chemotherapy

Patients suitable for treatment with a TPF induction chemotherapy includedthose with a good performance status, no contraindication to cisplatin or taxanes, andhigh tumor volume $\left(\mathrm{T}_{3} \mathrm{~T}_{4} \mathrm{~N}_{2} \mathrm{~N}_{3}\right)$. Other patients received(5 FU-CDDP) or (ADCDDP) regimens.

Tumor response to induction therapy was evaluated before commencement of RCT or RT by physical examination, nasopharyngoscopy (NP) and head and neck computer tomography (CT). Complete clinical response (CCR) was defined by a normal physical examination, normal NP and radiologic complete response. Radiologic response was assessed using computed tomography images based on Response Evaluation Criteria in Solid Tumors (RECIST). Then, 14 patients received concurrent chemoradiotherapy (RCT), 22 patients received radiotherapy (RT) alone. No patient received adjuvant chemotherapy. Three patients didn't receive neither RCT nor RT because of deterioration of general status . One patient had metastatic progression and received salvage chemotherapy.

Three dimensional (3D) conformal radiation therapy was used. Primitive tumor and clinically positive lymph nodes received $70 \mathrm{~Gy}$ and prophylactic neck lymph node levels were delivered to $50 \mathrm{~Gy}$ in 2 Gy/fraction, once a day, five times a week. After RCT,patients with a good performance status and good renal function received chemoradiotherapy .Patients with poor performance status or renal insufficiency received only radiotherapy. Schedules of RCT and RT are summarized in Table 2.

\begin{tabular}{|c|c|}
\hline Schedule & Dose \\
\hline chemoradiotherapy (RCT) & $\begin{array}{c}\text { weekly cisplatin } 40 \mathrm{mg} / \mathrm{m} 2 \\
\text { and RT : Primitive tumor and clinically positive lymph } \\
\text { nodes received 70 Gy and prophylactic neck lymp } \\
\text { node levels were delivered to } 50 \text { Gy in 2 Gy/fraction, } \\
\text { once a day, five times a week }\end{array}$ \\
\hline Only radiotherapy (RT) & $\begin{array}{c}\text { RT: Primitive tumor and clinically positive lymph } \\
\text { nodes received 70 Gy and prophylactic neck lymph } \\
\text { node levels were delivered to 50 Gy in 2 Gy/fraction, } \\
\text { once a day, five times a week }\end{array}$ \\
\hline
\end{tabular}

Table 2: Schedules of RCT and RT

\section{Results}

From January 2008 to December 2014, 40 patients were assessed. This study included 25 men and 15 women. The median age was 41 years. $37.5 \%$ of patients were diagnosed with stage IV. Patient Characteristics are detailed in Table 3.

\begin{tabular}{|l|l|l|}
\hline & \multicolumn{1}{|c|}{ N } & \multicolumn{1}{c|}{$\%$} \\
\hline $\begin{array}{l}\text { Sex } \\
\text { Male }\end{array}$ & 25 & 62.5 \\
\hline $\begin{array}{l}\text { Agemale } \text { Years } \\
\text { Median } \\
\text { Range }\end{array}$ & 15 & 37.5 \\
\hline $\begin{array}{l}\text { Median period of consultation } \\
\text { range }\end{array}$ & 41 \\
\hline
\end{tabular}




\begin{tabular}{|l|l|l|}
\hline & N & $\%$ \\
\hline UICC T-classification & & \\
T1 & 2 & 5 \\
T2 & 11 & 27 \\
T3 & 8 & 20 \\
T4 & 19 & 48 \\
\hline UICC N-classification & & \\
N0 & 8 & 20 \\
N1 & 7 & 18 \\
N2 & 10 & 25 \\
N3 & 15 & 37 \\
\hline M & & \\
M0 & 40 & 100 \\
M1 & 0 & 0 \\
\hline UICC Stage & & \\
II & 5 & 12.5 \\
III & 12 & 30 \\
IV A & 8 & 20 \\
IV B & 15 & 37,5 \\
\hline
\end{tabular}

Table 3: Patient Characteristics

Histologically, all tumors were undifferentiated carcinoma of nasopharyngeal 1 (type III WHO). Twenty patients received 5FUCDDP, 16 received TPF and 4 received AD-CDDP. The median number of cycles of chemotherapy was three (2-4 cycles).The median period of treatment initiation was 1,72 months (0-8 months). Responses after induction chemotherapyare detailed in Table 4.

\begin{tabular}{|c|c|c|c|c|}
\hline $\begin{array}{c}\text { Schedules of IC } \\
\text { Response after IC }\end{array}$ & 5FU-CDDP & TPF & AD-CDDP & Total \\
\hline Complete clinical response (CCR) & $9(45 \%)$ & $7(43.75 \%)$ & 2 & $18(45 \%)$ \\
\hline Partial clinical response (PR) & 10 & 9 & 2 & $21(52.5 \%)$ \\
\hline progression & 1 & 0 & 0 & $1(2.5 \%)$ \\
\hline Total & 20 & 16 & 4 & $40(100 \%)$ \\
\hline \multicolumn{4}{|c}{ Table 4: Response after IC }
\end{tabular}

In our study,the complete clinical response was higher in (5FU-CDDP) group ( $\mathrm{p}>0.05)$. The occurrence of leucopenia was higher in the 5FU-CDDP $(\mathrm{p}<0.05)$ group because patients received G-CSF in the TPF group. However, gastrointestinal toxicity was higher in the TPF group $(\mathrm{p}<0.05)$. Anemia and thrombopenia were similar between treatment groups. $11 \%$ of registered toxicities were Grade 4 (especially with 5FU-CDDP). After induction chemotherapy, 14 patients had RCT, 22 patients had only RT alone, 1 patient developed metastases and had salvage chemotherapy and 3 patients didn't have neither RCT nor RT because of deterioration of general status. Treatment after induction chemotherapy is detailed in Table 5.

\begin{tabular}{|c|c|c|c|c|c|}
\hline $\begin{array}{c}\text { Treatment } \\
\text { Response after IC }\end{array}$ & RCT & RT alone & $\begin{array}{c}\text { Salvage } \\
\text { chemotherapy }\end{array}$ & No treatment & Total \\
\hline CCR & 7 & 11 & 0 & 0 & 18 \\
\hline PR & 7 & 11 & 0 & 3 & 21 \\
\hline Progression & 0 & 0 & 1 & 0 & 1 \\
\hline Total & 14 & 22 & 1 & 3 & 40 \\
\hline
\end{tabular}

Table 5: Treatment after IC

In the RCT arm (14 patients), median number of chemotherapy cycles (weekly CDDP) was four (2-5 cycles). Primitive tumor and positive lymph nodes received $70 \mathrm{~Gy}$ and prophylactic neck lymph node levels were delivered to $50 \mathrm{~Gy}$ (2 Gy/fraction, once a day, five times a week).

In the RT arm (22 patients) median radiation dose of primitive tumor andpositive lymph nodes was 70 Gy andprophylactic neck lymph node levels were delivered to 50Gy. (2 Gy/fraction, once a day, five times a week). Complete response after RCT was $85 \%$ and $77 \%$ after RT. Responses after RCT and RT are summarized in Table 6.

\begin{tabular}{|c|c|c|c|c|}
\hline & \multicolumn{2}{|c|}{ CCR after IC } & \multicolumn{2}{c|}{ PR after IC } \\
\hline Treatment after IC & RCT & RT & RCT & RT \\
\hline N & 7 & 11 & 7 & 11 \\
\hline Response & Remission: 6 (85\%) & Remission:8 (73\%) & Remission: 6 (85\%) & Remission :9(82\%) \\
& Recurrence:1(15\%) & Recurrence:3(27\%) & Recurrence:1(15\%) & Recurrence 2(18\%) \\
\hline
\end{tabular}

Table 6: Response to RCT and RT 
Complete clinical response after induction chemotherapy followed by RCT was associated to better local control than RT alone. (85\%vs 73\%). Response to RCT was similar in the CCR and PR groups. However RT was associated to better local control in PR group than CCR group. The median overall survival in the complete clinical response group was: 38 months and was 30 months in partial response group. There was no benefit of overall survival in the CCR group compared to PR. ( $p=0.391$ ).Comparison of OS between complete clinical response and partial response groups is showed in Figure 1.

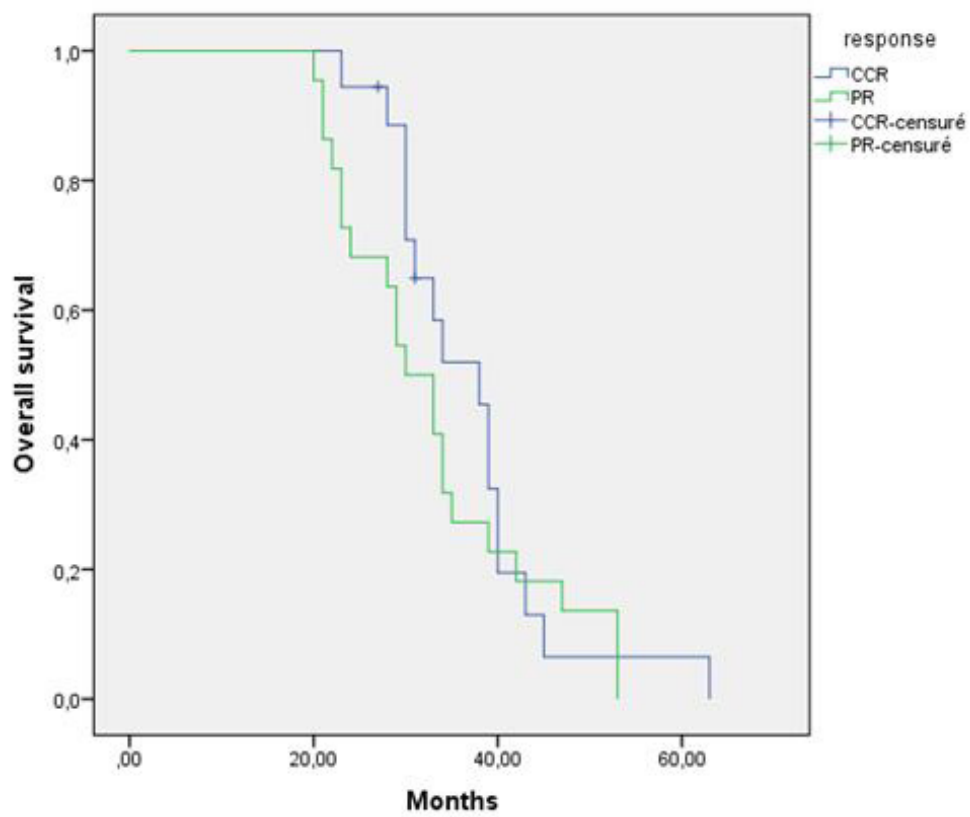

Figure 1: Comparison of overall survival between complete clinical response and partial response groups

\section{Discussion}

Tunisia is an area of intermediate incidence of nasopharyngeal carcinoma. (1-6 cases /100000) [6]. Therefore, defining the optimal treatment for patients with nasopharyngeal carcinoma is of the utmost importance. Treatment has substantially evolved in the last two decades. Current standard therapy for locally advanced (LA) nasopharyngeal carcinoma (NPC) is platinumbased concurrent chemoradiation. Although many trials demonstrated a benefit, induction chemotherapy is not a standard of care. Therefore, the effective population size of this study is relatively small. The majority of patients with LA-NPC in Tunisia received chemoradiation.

Epstein-Barr virus (EBV) has been long recognized as an etiologic agent of nasopharyngeal carcinoma [7]. Moreover, Many studies considered human papilloma virus (HPV) as a cause for NPC [8]. In our study, EBV status was accessed in few number of patients making data unexpoitable.We didn't access human papillomavirus status (HPV). Until mid-1990, RT was the standard treatment for all stages of NPC. Definitive radiation without chemotherapy was associated to a significant risk of local recurrences [9].

In 1998, the phase III randomized intergroup study 0099 compared chemoradiotherapy to radiotherapy alone in patients with nasopharyngeal cancers. The investigational arm received chemotherapy with cisplatin $100 \mathrm{mg} / \mathrm{m}^{2}$ on days 1,22 , and 43 during radiotherapy; postradiotherapy, chemotherapy with cisplatin $80 \mathrm{mg} / \mathrm{m}^{2}$ on day 1 and fluorouracil $1,000 \mathrm{mg} / \mathrm{m}^{2} / \mathrm{d}$ on days 1 to 4 was administered every 4 weeks for three cycles. This trial concluded that chemoradiotherapy is superior to radiotherapy alone for patients with advanced nasopharyngeal cancers with respect to PFS and overall survival [10]. Another Phase III randomized trial conducted in Singapore between September 1997 and May 2003 showed that RCT followed by adjuvant chemotherapy in LA-NPC improved significantly distant metastasis control, disease -free-survival (DFS) and overall survival (OS) compared to RT alone [11].

A systematic review and meta-analysis of randomized trials (1753 patients) conducted in 2006 by Baujat and al compared cisplatin-based chemotherapy plus RT with RT alone in locoregionally advanced NPC. This meta-analysis concluded that patients receiving any combined modality therapy (neoadjuvant, concurrent, or adjuvant chemotherapy) have an absolute event-free-survival (EFS) and OS benefit with the highest benefit resulting from RCT (The pooled hazard ratio of death was 0.82 (95\% confidence interval, $0.71-0.94 ; \mathrm{p}=0.006$ and pooled hazard ratio of tumor failure or death was 0.76 (95\% confidence interval, 0.67-0.86; $\mathrm{p}<0.0001$ ) [12]. Radio-chemotherapy was consideredthe standard of care as is the case in Tunisia. In order to improve survival and disease control, many trials evaluated the induction chemotherapy followed by RCT. Toxicity was also assessed.These studies produced mixed results. 
In 2004 ,ameta-analysis of Langendijk and al concluded that the addition of neoadjuvant chemotherapy to radiation resulted in a significant reduction ( $\mathrm{P}=0.005)$ of the incidence of locoregional recurrences (relative risk : $\mathrm{RR}, 0.74 ; 95 \% \mathrm{CI}, 0.60$ to 0.91 ) and significant reduction ( $\mathrm{P}=0.0003$ ) of the incidence of distant metastases, with an $\mathrm{RR}$ of 0.67 (95\% CI, 0.54 to 0.83 ) without OS benefit $(\mathrm{p}=0.13)$. There was no benefit to add adjuvant chemotherapy [13].

The study of J L OH and al demonstrated that IC followed by RCT in LA NPC (3cycles of IC consisting of cisplatin, 5FU, leucovorin and interferon- $a 2 b$ were administered, followed by RCT consisting of 7 cycles of 5-FU, hydroxyurea and oncedaily RTon a week-on week-off schedule) resulted in excellentoverall survival at 3 and 5 years (respectively 88\% and 77\%). Progression-free survival (PFS) at 3 and 5 years was respectively $92 \%$ and $86 \%$ with acceptable toxicity .After IC, there were $54.2 \%$ of complete response and $45.8 \%$ of partial response.After RCT , there was $100 \%$ of complete response. At 5 years, actuarial locoregional control was $93 \%$ and actuarial distant control 92\% [14].

In a randomized phase II trial, Hui et al. reported that neoadjuvant docetaxel-cisplatin followed by RCT provided a 3-year overall survival benefit in stage III-IVB NPC compared to RCT alone(94.1\% vs $67.7 \%, \mathrm{p}=0.012)$. Acute and late toxicities and quality of life scores were comparable [15]. In our series, complete response after induction chemotherapy was $45 \%$ versus $54,2 \%$ in the series of J L OH et al. RCT offered more remission than RT alone (85\% VS 77\%) which is similar to previous studies. The type of response after IC didn't affect OS. In contrast, there are negative studies. A greek phase II trial randomized 141 patients with LA-NPC to either 3 cycles of IC with cisplatin, epirubicin, and paclitaxel followed by definitive RT with concurrent weekly cisplatin versus RCT alone. There was no difference in the number of patients who completed radiation. There was no significant difference between the two treatment groups in OS (67\% versus $72 \%, \mathrm{p}=0.65)$ and PFS $(65 \%$ versus $64 \%, \mathrm{p}=0.71)[16]$.

The trial NPC-0501 evaluated the therapeutic gain by changing from concurrent-adjuvant to induction-Concurrent chemoradiotherapy, changing From Fluorouracil to Capecitabine, and changing from conventional to accelerated radiotherapy fractionation in patients with LA- NPC.Preliminary results indicated that the benefit of changing to an induction-concurrent sequence remains uncertain. Replacing fluorouracil with oral capecitabine warrants further validation in view of its convenience, favorable toxicity profile, and favorable trends in efficacy.Accelerated fractionation was not recommended for patients with locoregionally advanced NPC who receive chemoradiotherapy [17]. In our studythree regimens of IC were used. In the literature many trials compared different molecules and protocols of induction chemotherapyfollowed by RCT.The efficacy of docetaxel-cisplatin (TP) as induction chemotherapy regimen on NPC was similar to that of 5 -fluoruracil -cisplatin (PF) regimen, and the adverse events are tolerable [18].

Han SH and al demonstrated that the efficacy of vinorelbine-cisplatin (NP) regimen induction chemotherapy plus concurrent chemordiotherapy for advanced NPC is similar to that of TP regimen. The 3-year overall survival rates, disease-free-survival rates, locoregional relapse-free survival rates and distant metastasis-free survival rates in the NP and TP groups were $84.2 \%$ and $82.9 \%, 71.1 \%$ and $74.3 \%, 89.5 \%$ and $91.4 \%, 81.6 \%$ and $77.1 \%$, respectively $(\mathrm{P}>0.05)$. The toxicity of the NP regimen is lower than that of TP regimen [19]. In our study, NP regimen wasn't used. Several trials showed a benefit of TPF regimen.A phase II study showed that TPF induction chemotherapy followed by RCT had promising activity with manageable toxicity. The 3-year progression-free survival was $75.6 \%$ and the 3 -year overall survival was $86.1 \%$. [20]. According to Wen-Fei Li and al,the addition of TPF induction chemotherapy to RCTsignificantly increased failure-free survival, overall survival , and distant failure-free survival rates [21]. A Chinese study showed that IC followed by RCT was an effective treatment strategy for LANPC. Induction chemotherapy with TPF conferred satisfactory long-term survival and slightly improved PFS (77.0\% vs. 73.5\%; $\mathrm{P}=0.510)$ and $\mathrm{OS}(80.7 \%$ vs. $77.9 \%, \mathrm{P}=0.446)$ as compared with the classic PF regimen, and toxicity was tolerable [22].

In our study, patients who received induction 5FU -CDDP regimen achieved higher complete clinical response rate compared with that of patients receivingTPF regimen probably because there were more stage IVA - IVB NPC in the TPF group. In order to refine indications of induction chemotherapy, prognostic factors and risk stratification could help. Response to induction chemotherapy may have potential clinical value. Liu and al. revealed that the unsatisfactory tumor response after induction chemotherapy (stable disease or disease progression) could predict poor prognosis for patients with advanced-stage NPC (locoregional relapse-free survival,PFS) [23]. However ; an another trial concluded that the overall tumor response after induction chemotherapy was an independent prognostic factor for disease-free-survival, OS and locoregional recurrence free survival [24].

\section{Conclusion}

Induction chemotherapy followed by chemoradiation in LA-NCP carcinoma offers encouraging results. It doesn't be until today a standard of care. Complete clinical response after induction chemotherapycould be an interestingprognostic factor. This therapeutic stategy should be explored further in randomized settings in order to establish prognostic factors and to identify which patients benefit the most from IC/RCT. 


\section{References}

1. Boussen H, Bouaouina N (1999) Cancer du nasopharynx (cavum) EncyclMédChir. Oto-rhinolaryngologie, 20-590-A-10.

2. Boussen H, Bouaouina N, Mokni-Baizig N, A Gamoudi, L Chouchane et al. (2005). Carcinomes du nasopharynx: données actuelles. Pathol Biol 53: 45-51.

3. Golden DW, Rudra S, Witt ME, Nwizu T, Cohen EE et al. (2013) Outcomes of induction chemotherapy followed by concurrent chemoradiation for nasopharyngeal carcinoma. Oral Oncol 49:277-82.

4. Hao Peng, Lei Chen, Jian Zhang, Wen-Fei Li,Yan-Ping Mao et al. (2017) Induction Chemotherapy Improved Long-term Outcomes of Patients with Locoregionally Advanced Nasopharyngeal Carcinoma: A Propensity Matched Analysis of 5-year Survival Outcomes in the Era of Intensity-modulated Radiotherapy J cancer 8: 371-7.

5. Pu-Yun OuYang, Zhuo-Fei Bi, Lu-Ning Zhang, Kai-Yun You et al. (2016) Outcomes of Induction Chemotherapy Plus Intensity Modulated Radiotherapy (IMRT) Versus IMRT Plus Concurrent Chemotherapy for Locoregionally Advanced Nasopharyngeal Carcinoma: A Propensity Matched Study. Transl Oncol 9: 329-35.

6. Karray H, Ayadi W, Fki L, Hammami A, Daoud J et al. (2005) Comparison of three different serological techniques for primary diagnosis and monitoring of nasopharyngeal carcinoma in two age groups from Tunisia. J. Med virol 75: 593-602.

7. Mould RF, Tai TH (2002) Nasopharyngeal carcinoma: treatments and outcomes in the 20th century. Br J Radiol 75: 307-9.

8. Singhi AD, Califano J, Westra WH (2012) High-risk human papillomavirus in nasopharyngeal carcinoma. Head Neck 34: 213-18.

9. Cooper JS, del Rowe J, Newall J (1983) Regional Stage IV carcinoma of the nasopharynx treated by aggressive radiotherapy. Int J Radiat Oncol Biol Phys 9: 1737-45.

10. Al-Sarraf M, Leblanc M, Giri S, Fu KK, Cooper J et al. (1998) Chemoradiotherapy versus radiotherapy in patients with advanced nasopharyngeal cancer: phase III randomized Intergroup Study 0099. J Clin Oncol 16: 1310-7.

11. Wee J1, Tan EH, Tai BC, Wong HB, Leong SS et al. (2005) Randomized trial of radiotherapy versus concurrent chemoradiotherapy followed by adjuvant chemotherapy in patients with American Joint Committee on Cancer/International Union against cancer stage III and IV nasopharyngeal cancer of the endemic variety. J Clin Oncol 23: 6730-8.

12. Baujat B, Audry H, Bourhis J, Chan AT, Onat H et al. (2006) Chemotherapy in locally advanced nasopharyngeal carcinoma: an individual patient data metaanalysis of eight randomized trials and 1753 patients. Int J Radiat Oncol Biol Phys 64: 47-56.

13. Langendijk JA, Leemans CR, Buter J, Berkhof J, Slotman BJ (2004) The additional value of chemotherapy to radiotherapy in locally advanced nasopharyngeal carcinoma: a meta-analysis of the published literature. J Clin Oncol 22: 4604-12.

14. Oh JL, Vokes EE, Kies MS, Mittal BB, Witt ME et al. (2003) Induction chemotherapy followed by concomitant chemoradiotherapy in the treatment of locoregionally advanced nasopharyngeal cancer. Ann Oncol 14: 564 - 69.

15. Hui EP, Ma BB, Leung SF, King AD, Mo F et al. (2009) Randomized phase II trial of concurrent cisplatin-radiotherapy with or without neoadjuvantdocetaxel and cisplatin in advanced nasopharyngeal carcinoma. J Clin Oncol 27: 242-9.

16. Fountzilas G, Ciuleanu E, Bobos M, Kalogera-Fountzila A, Eleftheraki AG et al. (2012) Induction chemotherapy followed by concomitant radiotherapy and weekly cisplatin versus the same concomitant chemoradiotherapy in patients with nasopharyngeal carcinoma: a randomized phase II study conducted by the Hellenic Cooperative Oncology Group (HeCOG) with biomarker evaluation. Ann Oncol 23:427-35.

17. Lee AW, Ngan RK, Tung SY, Cheng A, Kwong DL et al. (2015) Preliminary results of trial NPC-0501 evaluating the therapeutic gain by changing from concurrent-adjuvant to induction-concurrent chemoradiotherapy, changing from fluorouracil to capecitabine, and changing from conventional to accelerated radiotherapy fractionation in patients with locoregionally advanced nasopharyngeal carcinoma. Cancer 121: 1328-38.

18. Xie FY, Qi SN, Hu WH, Zou GR, Peng M et al. (2007) Comparison of efficacy of docetaxel combined cisplatin (TP regimen) and cisplatin combined 5-fluorouracil (PF regimen) on locally advanced nasopharyngeal carcinoma. Ai Zheng 26: 880-4.

19. Han SH, Yu L, Zhang Z, Zhang PJ, Song HP et al (2013) Evaluation of induction chemotherapy with vinorelbine plus cisplatin (NP) or docetaxel plus cisplatin (TP) combined with concurrent chemoradiotherapy for patients with locally advanced nasopharyngeal carcinoma. Zhonghua Zhong Liu Za Zhi 35: 623: 6 .

20. Bae WK, Hwang JE, Shim HJ, Cho SH, Lee JK et al. (2010) Phase II study of docetaxel, cisplatin, and 5-FU induction chemotherapy followed by chemoradiotherapy in locoregionally advanced nasopharyngeal cancer. Cancer Chemother Pharmacol 65: 589-95.

21. Wen-Fei Li, Lei Chen, Ying Sun, Jun Ma (2016) Induction chemotherapy for locoregionally advanced nasopharyngeal carcinoma. Chin J Cancer 35: 94.

22. Zeli Huang, Jiezhan Feng, Shaoen Li, Weihong Wei, Guoyi Zhang et al. (2013) Is the Neoadjuvant Docetaxel, Cisplatin and 5-Fluorouracil regimen Superior to Classic Cisplatin and 5-Fluorouracil for locoregionally Advanced Nasopharyngeal Carcinoma? J Cancer Res Upd 2: 297-305.

23. Liu LT, Tang LQ, Chen QY, Zhang L, Guo SS et al. (2015) The Prognostic Value of Plasma Epstein-Barr Viral DNA and Tumor Response to Neoadjuvant Chemotherapy in Advanced-Stage Nasopharyngeal Carcinoma. Int J Radiat Oncol Biol Phys 93: 862-9.

24. Hao Peng, Lei Chen, Yuan Zhang, Wen-Fei Li, Yan-Ping Mao (2016) The Tumour Response to Induction Chemotherapy has Prognostic Value for Long-Term Survival Outcomes after Intensity-Modulated Radiation Therapy in Nasopharyngeal Carcinoma. Sci Rep 6: 24835. 


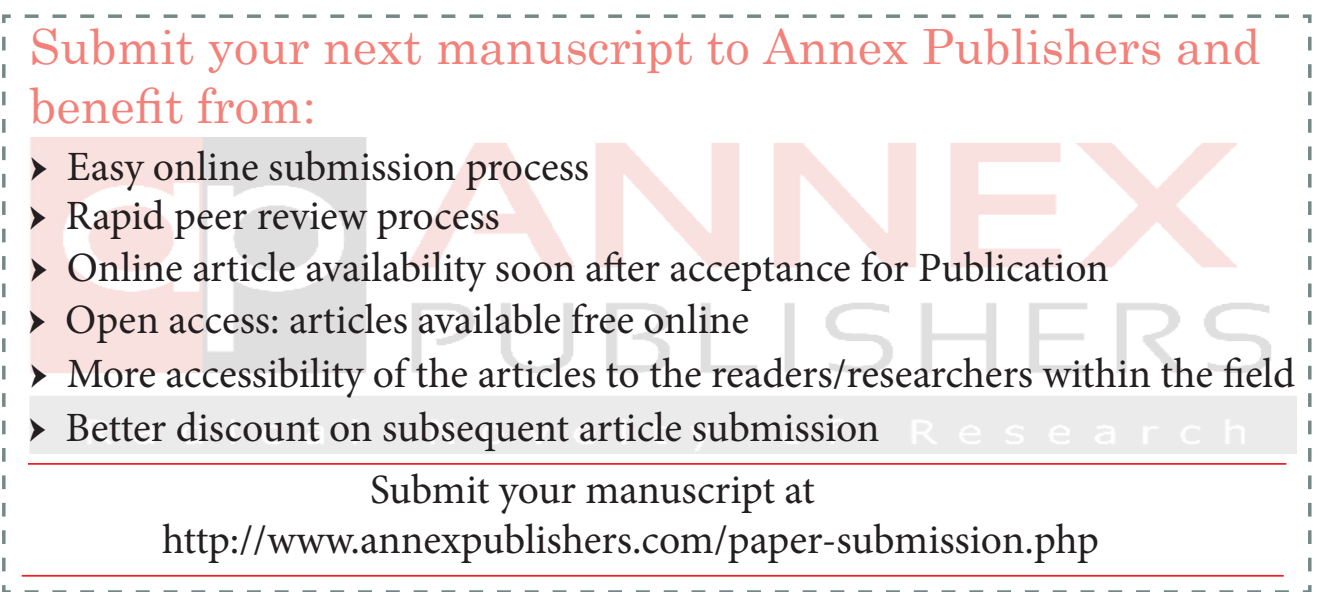

